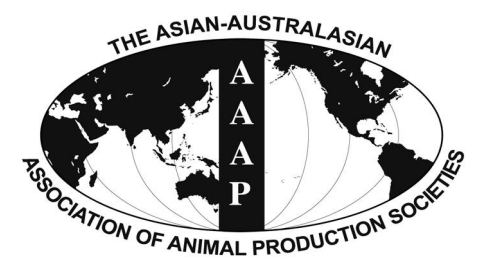

Open Access

Asian Australas. J. Anim. Sci.

Vol. 28, No. 12 : 1713-1720 December 2015

http://dx.doi.org/10.5713/ajas.15.0095

www.ajas.info

pISSN 1011-2367 elSSN 1976-5517

\title{
The Usefulness of Selected Physicochemical Indices, Cell Membrane Integrity and Sperm Chromatin Structure in Assessments of Boar Semen Sensitivity
}

\author{
A. Wysokińska*, S. Kondracki, and M. Iwanina \\ Institute of Bioengineering and Animal Breeding, Faculty of Natural Science, \\ Siedlce University of Natural Sciences and Humanities, 08-110 Siedlce, Poland
}

\begin{abstract}
The present work describes experiments undertaken to evaluate the usefulness of selected physicochemical indices of semen, cell membrane integrity and sperm chromatin structure for the assessment of boar semen sensitivity to processes connected with pre-insemination procedures. The experiments were carried out on 30 boars: including 15 regarded as providers of sensitive semen and 15 regarded as providers of semen that is little sensitive to laboratory processing. The selection of boars for both groups was based on sperm morphology analyses, assuming secondary morphological change incidence in spermatozoa as the criterion. Two ejaculates were manually collected from each boar at an interval of 3 to 4 months. The following analyses were carried out for each ejaculate: sperm motility assessment, sperm $\mathrm{pH}$ measurement, sperm morphology assessment, sperm chromatin structure evaluation and cell membrane integrity assessment. The analyses were performed three times. Semen storage did not cause an increase in the incidence of secondary morphological changes in the group of boars considered to provide sperm of low sensitivity. On the other hand, with continued storage there was a marked increase in the incidence of spermatozoa with secondary morphological changes in the group of boars regarded as producing more sensitive semen. Ejaculates of group I boars evaluated directly after collection had an approximately $6 \%$ smaller share of spermatozoa with undamaged cell membranes than the ejaculates of boars in group II ( $\leq \leq 0.05)$. In the process of time the percentage of spermatozoa with undamaged cell membranes decreased. The sperm of group I boars was characterised with a lower sperm motility than the semen of group II boars. After 1 hour of storing diluted semen, the sperm motility of boars producing highly sensitive semen was already $4 \%$ lower $(\mathrm{p} \leq 0.05)$, and after 24 hours of storage it was $6.33 \%$ lower than that of the boars that produced semen with a low sensitivity. Factors that confirm the accuracy of insemination male selection can include a low rate of sperm motility decrease during the storage of diluted semen, low and contained incidence of secondary morphological changes in spermatozoa during semen storage and a high frequency of spermatozoa with undamaged cell membranes. (Key Words: Boar, Chromatin Structure, Sperm Membrane Integrity, Sperm Morphology)
\end{abstract}

\section{INTRODUCTION}

Analyses of the male reproductive potential constitute an important and difficult task in insemination practice. They require constant improvements of techniques for assessing semen obtained from sires. Standard semen assessment includes the analysis of basic physical parameters of the ejaculate and is performed directly

\footnotetext{
* Corresponding Author: A. Wysokińska. Tel: +48-25-6431388, E-mail: annaw@uph.edu.pl

Submitted Feb. 3, 2015; Revised Apr. 11, 2015; Accepted May 8, 2015
}

following ejaculate collection. The assessment of ejaculate parameters is generally a subjective evaluation and provides not enough information on the processes taking place in spermatozoa and semen plasma. The spermatozoon is one of the most differentiated cells in mammalian organisms. It is highly sensitive to the effects of external factors. Ejaculate processing after its collection can cause changes in sperm cell structures, thus affecting sperm survival rate and fertilisation capacity (Knox and Yantis, 2014). It is not a common insemination practice to analyse the changes that may occur in semen following its collection and dilution. However, factors that are at work during dilution, 
preservation and storage of diluted semen can generate structural changes within spermatozoa (Manee-In et al., 2014; Wysokińska and Kondracki, 2014). Apart from traditional semen assessment methods, ejaculate analyses increasingly more often involve other analytic techniques that make it possible to obtain precise information on the structure and functioning of spermatozoa and the appearance of pathological changes in sperms which can affect egg cell fertilisation (Waberski et al., 2008). The efficacy of egg cell penetration depends on the activity and vitality of spermatozoa and the functioning of their locomotor system (Kwon et al., 2014). The survival rate of spermatozoa is conditioned by the integrity of cell membranes and metabolic processes. Hence, new methods are being sought to provide information on the functioning of spermatozoa outside the male organism and on factors that influence sperm capacity for fertilisation (Park et al., 2012). It is important that the methods should be inexpensive, easily practicable and possible to include in insemination practice.

Boar fitness for reproduction depends on the breeding value and ejaculatory performance of the male (Kawęcka et al., 2008). Particular animals can, however, significantly differ as to the sensitivity of their sperm to processes occurring during semen preservation, storage and handling. This can substantially differentiate the predisposition of particular males for insemination use. The present work describes experiments undertaken to evaluate the usefulness of selected physicochemical indices of semen, cell membrane integrity and sperm chromatin structure for the assessment of boar semen sensitivity to processes connected with pre-insemination procedures.

\section{MATERIALS AND METHODS}

\section{Biological materials and semen assessment}

The experiments were carried out on 30 insemination boars in age from 1.5 to 2 years. The qualification of boars to the experimental groups was based on sperm morphology analyses of at least 5 ejaculates previously collected from each boar. The selection of boars for groups was based on sperm morphology analyses, assuming secondary morphological change incidence in spermatozoa as the criterion according to Blom's classification (Blom, 1981). The group of boars considered to be providers of sensitive semen included sires whose ejaculates contained at least $10 \%$ of spermatozoa with secondary morphological changes. On the other hand, the group of boars producing low sensitive semen included sires whose ejaculates contained negligible numbers of spermatozoa with secondary morphological changes, with the incidence rate of up to $5 \%$ in each case. The groups created in this way were used in a strictly controlled experiment. Two ejaculates were manually collected from each boar at an interval of 3 to 4 months. The following analyses were carried out for each ejaculate: sperm motility assessment, sperm $\mathrm{pH}$ measurement, sperm morphology assessment, sperm chromatin structure evaluation and cell membrane integrity assessment. The analyses were performed three times, i.e., directly after ejaculate collection, after dilution (1 hour from collection) and after 24 hours of storage of processed semen at $17^{\circ} \mathrm{C}$. The ejaculates were diluted in the Cronos diluent (Medinowa, Reggio Emilia, Italy) warmed to $37^{\circ} \mathrm{C}$ and subsequently stored in a thermally insulated box at $17^{\circ} \mathrm{C}$.

\section{Sperm morphology and motility evaluation}

Sperm motility was evaluated with a Nikon Eclipse 50i light microscope equipped with a heated stage. A $5 \mu \mathrm{L}$ of sperm suspension was put a pre-warmed slide and then covered with a coverslip at $37^{\circ} \mathrm{C}$. At a 200 -fold zoom we determined the percentage of correctly motile spermatozoa in the overall number of sperms present in the field of vision of the microscope. Sperm morphology was assessed based on the results of the microscopic analysis of preparations made from the samples of all collected ejaculates. The preparations intended for morphological analyses were made according to the eosin-gentian dye method (Kondracki et al., 2012). The microscopic analyses of the preparations were performed with 100-fold zoom immersion lenses on a Nikon Eclipse 50i light microscope. The morphological structure of 500 spermatozoa was assessed in each preparation, identifying the number of well-formed and morphologically altered spermatozoa and differentiating those with primary and secondary changes according to Blom's classification (Blom, 1981).

\section{Assessment of sperm chromatin structure}

The chromatin structure of the spermatozoa was evaluated using acridine orange according to a methodology proposed by Bochenek et al. (2001). $0.4 \mathrm{~mL}$ of the solution A (Triton X-100, $0.1 \mathrm{~mL}$; $\mathrm{HCl} 1.0 \mathrm{~N}, 8 \mathrm{~mL}$; NaCl, $0.877 \mathrm{~g}$; $\mathrm{H}_{2} \mathrm{O} 2 \times$ distilled) was added to $0.2 \mathrm{~mL}$ of a sperm (concentration $1 \times 10^{6} / \mathrm{mL}$ spermatozoa) and was incubated in a temperature of $4^{\circ} \mathrm{C}$ for 30 seconds. Thereafter, $1.2 \mathrm{~mL}$ of the solution B (Citric-phosphate Buffet, $100 \mathrm{~mL}$; NaCl, $0.877 \mathrm{~g} ; \mathrm{Na}_{2}$ EDTA, $34 \mathrm{mg}$; Acridine orange $[1 \mathrm{mg} / \mathrm{mL}$ $\mathrm{H} 2 \mathrm{O}$ ], $0.6 \mathrm{~mL}$ [Sigma-Aldrich, St. Louis, MO, USA]) was added and incubated in a temperature of $4^{\circ} \mathrm{C}$ for 3 minutes. Two hundred spermatozoa were analysed in each preparation, specifying sperms with a correct chromatin structure (sperm heads emitting green fluorescence) and sperms with an incorrect chromatin structure (sperm heads emitting orange fluorescence). Sperm chromatin structure was assessed using a Nikon Eclipse 50i microscope with a 
fluorescence attachment.

\section{Assessment of sperm cell membrane integrity}

The preparations for analysing cell membrane integrity were made using two methods.

The SYBR-14/PI staining method: The preparations were stained using the Live/Dead Sperm Viability Kit (Molecular Probes Inc., Leiden, Netherlands). The preparation was made according to the following methodology: $1 \mathrm{~mL}$ of diluted ejaculate was supplemented with $5 \mu \mathrm{L}$ of SYBR-14 diluted 50 -fold in deionised water and incubated at $36^{\circ} \mathrm{C}$ for 10 minutes (concentration 100 $\mathrm{nM}$ ); subsequently, $5 \mu \mathrm{L}$ propidium iodide (PI) (concentration $12 \mu \mathrm{m}$ ) was added and the preparation was incubated for 10 minutes at $36^{\circ} \mathrm{C}$. A drop of the solution was placed on a slide preheated to $37^{\circ} \mathrm{C}$ and a sperm cell membrane integrity analysis was performed using a Nikon Eclipse 50i microscope equipped with a fluorescence analysis adapter (filter B-2EC, TRITC). The cell membrane integrity assessment was made directly after the samples were prepared. Two hundred spermatozoa were analysed in each preparation. The following groups of spermatozoa were distinguished: those emitting green fluorescence over the entire area of the head were designated as living cells (with an intact cell membrane, stained with SYBR 14); the spermatozoa that emitted red fluorescence over the entire or part of the head area were designated as dead cells (with a damaged cell membrane, stained with PI); and the spermatozoa that exhibited yellow and orange fluorescence within the entire head area were identified as moribund sperm.

The eosin-nigrosin staining method (differential staining): The preparations for analyses were made according to the following methodology: a drop of semen was placed on a slide preheated to $40^{\circ} \mathrm{C}$ and mixed with twice the volume of the dye mixture (one part 5\% bluish eosin solution (Carl Roth Gmbh+Co. KG, Karlsruhe, Germany) to four parts $10 \%$ nigrosin aqueous solution [Sigma-Aldrich, USA]) using a glass rod to produce a smear on the slide. The samples were air-dried at room temperature. Two hundred spermatozoa were assessed in each preparation, again using the fluorescence microscopy techniques described previously. The stained spermatozoa were classified as those with a viable cell membrane structure (unstained/living) and those with a damaged membrane structure (pink-stained/dead).

\section{Statistical analysis}

Experimental data were analyzed using a program STATISTICA 10 PL (StatSoft, Tulsa, OK, USA). PL. All results are expressed as mean \pm standard deviation. The obtained material was statistically analysed according to the following mathematical model: $\mathrm{Y}_{i j}=\mu+\mathrm{a}_{i}+\mathrm{e}_{i j}$, where: $\mathrm{Y}_{i j}$, value of the analysed parameter; $\mu$, population mean; $\mathrm{a}_{i}$, boar semen sensitivity effect; $\mathrm{e}_{i j}$, error. The significance of the differences between the groups was assessed with the Tukey test at $\mathrm{p} \leq 0.05$ and $\mathrm{p} \leq 0.01$.

\section{RESULTS}

Changes in sperm motility that occurred during the storage of the sperm of boars regarded as providers of sensitive semen and those that produced semen resistant to processes taking place during ejaculate treatment presents in Table 1. The sperm of group I boars was characterised with a lower sperm motility than the sperm of group II boars. As expected, with elapsing time of storage we observed a fall in sperm motility in both analysed groups. However, sperm motility in group I decreased at a much higher rate than the motility of spermatozoa of boars in group II. After 1 hour of storing diluted semen, the sperm motility of boars producing highly sensitive semen was already $4 \%$ lower $(\mathrm{p} \leq 0.05)$, and after 24 hours of storage it was $6.33 \%$ lower than that of the boars that produced semen with a low sensitivity. Over the 24 hours of storage, sperm motility in group I diminished by almost $27 \%$, whereas the analogous decrease in group II was much smaller and amounted to approximately $21 \%$. It should be noted that along with storage time there was a gradual increase of the level of variability in sperm motility in both groups. This testifies to considerable individual differentiation, highlighting the importance of individual predispositions for insemination.

No significant differences were identified in the $\mathrm{pH}$ of

Table 1. Sperm motility changes (mean \pm standard deviation) during semen storage

\begin{tabular}{lcc}
\hline Item & $\begin{array}{c}\text { Group I } \\
\text { Number of boars }\end{array}$ & $\begin{array}{c}\text { Group II } \\
\text { (boars producing highly sensitive semen) }\end{array}$ \\
Number of ejaculates & 15 & 15 \\
Percentage of spermatozoa with progressive motility & 30 & 30 \\
$\quad$ Directly after collection (undiluted ejaculate) & & $77.67 \pm 5.68$ \\
After 1 hour from collection (diluted ejaculate) & $76.67 \pm 5.47$ & $76.33^{\mathrm{b}} \pm 7.06$ \\
After 24 hours from collection (diluted ejaculate) & $72.33^{\mathrm{a}} \pm 6.12$ & $56.33 \pm 17.26$ \\
\hline
\end{tabular}

Values within the same row followed by the different small letter superscripts are significantly different $(\mathrm{p} \leq 0.05)$. 
Table 2. The $\mathrm{pH}$ of the ejaculates of boars (mean \pm standard deviation)

\begin{tabular}{lcc}
\hline Item & $\begin{array}{c}\text { Group I } \\
\text { pH directly after collection (undiluted ejaculate) }\end{array}$ & $\begin{array}{c}\text { Group II } \\
\text { (boars producing highly sensitive semen) }\end{array}$ \\
pH after 1 hour from collection (diluted ejaculate) & $7.26 \pm 0.14$ & $7.24 \pm 0.17$ \\
pH after 24 hours from collection (diluted ejaculate) & $7.04 \pm 0.08$ & $7.02 \pm 0.08$ \\
\hline
\end{tabular}

the ejaculates in both analysed groups (Table 2). The ejaculates evaluated directly after collection had higher $\mathrm{pH}$ values than the diluted ejaculates assessed 1 hour from collection. After 24 hours of storage we noticed a slight rise in the semen $\mathrm{pH}$ levels. The $\mathrm{pH}$ changes observed during semen storage were however insignificant and had a similar course in both boar groups.

Table 3 contains the results of the sperm morphology analyses. The data presented show that the incidence of morphologically well-formed spermatozoa in the semen of boars regarded as providers of highly sensitive semen (group I) was lower than in the case of group II ejaculates already at collection. The difference was $5.4 \%(\mathrm{p} \leq 0.05)$. As semen storage continued, the percentage of morphologically well-formed spermatozoa fell. The changes were however insignificant in the group of boars that produced semen with low sensitivity (group II). On the other hand, we observed a dynamic fall in the number of morphologically well-formed spermatozoa in the group of boars that provided highly sensitive semen (group I). Hence, in the process of time the differences between the analysed groups increased. After 1 hour of storing diluted semen the incidence of morphologically well-formed spermatozoa in the group of boars with highly sensitive semen was already almost $11 \%$ lower than in the group of boars with more resistant sperm, and after 24 hours of storage the difference increased to approximately $12 \%(p \leq 0.01)$. The analysis of incidence of morphological changes in the spermatozoa produced interesting observations. With the process of time during storage the incidence of such changes rose. Semen storage did not cause an increase in the incidence of secondary morphological changes in the group of boars considered to provide sperm of low sensitivity. On the other hand, with continued storage there was a marked increase in the incidence of spermatozoa with secondary morphological changes in the group of boars regarded as producing more sensitive semen. Intergroup differences were relatively large, statistically significant and increased with continued semen storage.

The state of sperm chromatin structure is not significantly correlated with boar semen sensitivity defined on the basis of the frequency of secondary morphological changes in spermatozoa (Table 4). Around 99\% of spermatozoa in the ejaculates of boars in both groups had a correct chromatin structure. This was observed both in fresh and diluted ejaculates.

The incidence of spermatozoa with damaged and undamaged cell membrane, differentiated by means of propidium iodide and SYBR-14 staining presents in Table 5. Ejaculates of the sires regarded as providers of sensitive semen (group I) contained a lower percentage of spermatozoa with an undamaged cell membrane structure than the ejaculates of boars in group II. Ejaculates of group I boars evaluated directly after collection had an approximately $6 \%$ smaller share of spermatozoa with undamaged cell membranes than the ejaculates of boars in group II $(p \leq 0.05)$. In the process of time the percentage of spermatozoa with undamaged cell membranes decreased. It

Table 3. Incidence of morphologically well-formed and altered spermatozoa (means \pm standard deviation) in relation to semen sensitivity

\begin{tabular}{lcc}
\hline Item & $\begin{array}{c}\text { Group I } \\
\text { (boars producing highly sensitive semen) }\end{array}$ & $\begin{array}{c}\text { Group II } \\
\text { (boars producing low sensitive semen) }\end{array}$ \\
\hline Morphologically well-formed spermatozoa (\%) & & $94.59^{\mathrm{b}} \pm 4.12$ \\
Directly after collection (undiluted ejaculate) & $89.19^{\mathrm{a}} \pm 10.82$ & $94.70^{\mathrm{B}} \pm 4.29$ \\
After 1 hour from collection (diluted ejaculate) & $83.81^{\mathrm{A}} \pm 16.36$ & $91.51^{\mathrm{B}} \pm 10.52$ \\
After 24 hours from collection (diluted ejaculate) & $79.61^{\mathrm{A}} \pm 16.13$ & $1.46 \pm 1.15$ \\
Spermatozoa with primary changes (\%) & & $2.57 \pm 2.23$ \\
Directly after collection (undiluted ejaculate) & $1.80 \pm 1.76$ & $5.31 \pm 4.74$ \\
After 1 hour from collection (diluted ejaculate) & $2.13 \pm 2.10$ & $3.95^{\mathrm{B}} \pm 3.51$ \\
After 24 hours from collection (diluted ejaculate) & $4.76 \pm 4.04$ & $2.73^{\mathrm{B}} \pm 2.70$ \\
Spermatozoa with secondary changes (\%) & & $3.19^{\mathrm{B}} \pm 2.73$ \\
Directly after collection (undiluted ejaculate) & $9.01^{\mathrm{A}} \pm 8.55$ & $14.06^{\mathrm{A}} \pm 13.28$ \\
After 1 hour from collection (diluted ejaculate) & $15.63^{\mathrm{A}} \pm 11.42$ & \\
After 24 hours from collection (diluted ejaculate) & & \\
\hline
\end{tabular}

Different superscripts mean significant differences among means within particular rows (lower-case letters, $\mathrm{p} \leq 0.05$; upper-case letters, $\mathrm{p} \leq 0.01$ ). 
Table 4. Incidence of spermatozoa with a correct and damaged chromatin structure (means \pm standard deviation)

\begin{tabular}{lcc}
\hline Item & $\begin{array}{c}\text { Group I } \\
\text { Spermatozoa with a correct chromatin structure (\%) }\end{array}$ & $\begin{array}{c}\text { Group II } \\
\text { (boars producing highly sensitive semen) }\end{array}$ \\
Directly after collection (undiluted ejaculate) & & $99.13 \pm 0.30$ \\
After 1 hour from collection (diluted ejaculate) & $99.11 \pm 0.31$ & $99.15 \pm 0.32$ \\
After 24 hours from collection (diluted ejaculate) & $98.68 \pm 0.48$ & $99.13 \pm 0.27$ \\
Spermatozoa with an incorrect chromatin structure (\%) & $99.07 \pm 0.33$ & $0.87 \pm 0.30$ \\
Directly after collection (undiluted ejaculate) & & $0.85 \pm 0.32$ \\
After 1 hour from collection (diluted ejaculate) & $0.89 \pm 0.31$ & $0.87 \pm 0.27$ \\
After 24 hours from collection (diluted ejaculate) & $1.32 \pm 0.48$ & $0.93 \pm 0.33$ \\
\hline
\end{tabular}

seems, however, that the rate of the changes was slightly slower in the group of boars producing highly sensitive semen. As a result, after 24 hours of storage intergroup differences diminished to a level that did not exceed $1 \%$ $(\mathrm{p}>0.05)$. Similar conclusions were drawn from the analysis of incidence of spermatozoa with damaged cell membranes, identified as dead, and those identified as moribund. In both cases the observed intergroup differences were the greatest directly after ejaculate collection and diminished in the process of time with continued semen storage. The significance of intergroup differences was confirmed in the case of moribund spermatozoa in the analysis conducted directly after ejaculate collection. The incidence of moribund spermatozoa was then $3.88 \%$ higher in the ejaculates of boars the produced more sensitive semen $(\mathrm{p} \leq 0.05)$.

The data that describe the incidence of spermatozoa with damaged and undamaged cell membranes, differentiated by means of eosin-nigrosine staining presents in Table 6. The data reveal that boars providing sensitive semen produce more spermatozoa with a damaged cell membrane structure and fewer sperms with undamaged cell membranes than the boars in group II. Such tendencies were observed both in the results of the assessment of fresh ejaculates and in the diluted ejaculates evaluated after 1 and 24 hours of storage. The observed differences were not however confirmed statistically. It was observed, in turn, that in the group of boars regarded as providers of sensitive semen the number of sperms with a damaged cell membrane structure rose immediately after ejaculate dilution, in contrast to group II. This shows that it is possible to take advantage of the incidence of spermatozoa with a damaged cell membrane structure in collected ejaculates as the criterion for the assessment of sperm sensitivity to laboratory processing.

\section{DISCUSSION}

The data of the present work reveal that semen collected form particular boars exhibits substantial variability in the degree of sensitivity to pre-insemination treatment procedures, especially those connected with dilution and storage of diluted sperm prior to performing the insemination intervention. Variability in the fitness of ejaculates for insemination use constitutes a serious practical problem (Broekhuijse et al., 2012).

Table 5. Incidence of spermatozoa with damaged and undamaged cell membranes (propidium iodide and SYBR-14 staining) (mean \pm standard deviation)

\begin{tabular}{|c|c|c|}
\hline Item & $\begin{array}{c}\text { Group I } \\
\text { (boars producing highly sensitive semen) }\end{array}$ & $\begin{array}{c}\text { Group II } \\
\text { (boars producing low sensitive semen) }\end{array}$ \\
\hline \multicolumn{3}{|l|}{ Spermatozoa with undamaged cell membranes (live) (\%) } \\
\hline Directly after collection (undiluted ejaculate) & $85.80^{\mathrm{A}} \pm 13.54$ & $91.82^{\mathrm{B}} \pm 5.07$ \\
\hline After 1 hour from collection (diluted ejaculate) & $83.13 \pm 12.38$ & $87.66 \pm 5.19$ \\
\hline After 24 hours from collection (diluted ejaculate) & $77.63 \pm 16.74$ & $78.43 \pm 17.15$ \\
\hline \multicolumn{3}{|l|}{ Spermatozoa with damaged cell membranes (dead) (\%) } \\
\hline Directly after collection (undiluted ejaculate) & $7.40 \pm 7.15$ & $5.27 \pm 3.35$ \\
\hline After 1 hour from collection (diluted ejaculate) & $11.12 \pm 8.24$ & $8.13 \pm 4.65$ \\
\hline After 24 hours from collection (diluted ejaculate) & $15.05 \pm 11.37$ & $14.52 \pm 9.79$ \\
\hline \multicolumn{3}{|l|}{ Moribund spermatozoa (\%) } \\
\hline Directly after collection (undiluted ejaculate) & $6.80^{\mathrm{a}} \pm 6.48$ & $2.92^{\mathrm{b}} \pm 2.79$ \\
\hline After 1 hour from collection (diluted ejaculate) & $5.75 \pm 4.73$ & $4.20 \pm 4.12$ \\
\hline After 24 hours from collection (diluted ejaculate) & $7.31 \pm 6.64$ & $7.05 \pm 6.74$ \\
\hline
\end{tabular}

Different superscripts mean significant differences among means within particular rows (lower-case letters, $\mathrm{p} \leq 0.05$; upper-case letters, $\mathrm{p} \leq 0.01$ ). 
Table 6. Incidence of spermatozoa with damaged and undamaged cell membranes, differentiated by means of eosin-nigrosine staining (means \pm standard deviation)

\begin{tabular}{lcc}
\hline Item & $\begin{array}{c}\text { Group I } \\
\text { (boars producing highly sensitive semen) }\end{array}$ & $\begin{array}{c}\text { Group II } \\
\text { (boars producing low sensitive semen) }\end{array}$ \\
\hline $\begin{array}{l}\text { Spermatozoa with undamaged cell membranes (live) (\%) } \\
\quad \text { Directly after collection (undiluted ejaculate) }\end{array}$ & $84.03 \pm 13.83$ & $87.80 \pm 10.80$ \\
After 1 hour from collection (diluted ejaculate) & $82.80 \pm 14.51$ & $87.61 \pm 11.76$ \\
After 24 hours from collection (diluted ejaculate) & $80.00 \pm 16.44$ & $86.15 \pm 13.14$ \\
Spermatozoa with damaged cell membranes (dead) (\%) & & $12.20 \pm 10.80$ \\
Directly after collection (undiluted ejaculate) & $15.97 \pm 13.83$ & $12.38 \pm 11.76$ \\
After 1 hour from collection (diluted ejaculate) & $17.20 \pm 14.51$ & $13.85 \pm 13.14$ \\
After 24 hours from collection (diluted ejaculate) & $19.63 \pm 15.37$ & \\
\hline
\end{tabular}

We demonstrated in the present study that semen sensitivity to the action of external pre-insemination factors is an individual trait of boars and it is possible to select males with a particular fitness for insemination service. We also demonstrated that the incidence of secondary morphological changes in spermatozoa, defined at the beginning of insemination service of a given boar, is a simple and practically feasible index of boar semen sensitivity. Secondary morphological changes come about after the development of the main sperm structure in semen outlet ducts and after discharging the ejaculate by the male (Briz et al., 1996). The incidence of secondary morphological changes in spermatozoa depends on the accuracy of dealing with semen in laboratory processing and during its storage prior to insemination use (Oberlender et al., 2012). Any mistakes made in this respect lead to an increase in the incidence of secondary morphological changes. The data of the present study clearly show that such sensitivity exists and the incidence of secondary morphological changes in spermatozoa can be an effective tool in the assessment of boar fitness for insemination service.

The data of the present work reveal that sperm motility can be an index of the accuracy of selection of males for insemination. This is confirmed by the pattern of sperm motility decrease during the storage of diluted semen, much slower in the ejaculates of boars producing semen of low sensitivity. Prolonged semen storage is usually accompanied with a deterioration of sperm quality (MartínHidalgo et al., 2013). De Ambrogi et al. (2006) have observed that after 72 hours of semen storage sperm motility significantly declines. The motility of spermatozoa in diluted semen can deteriorate as a result of temperature changes (López Rodríguez et al., 2012). It has been demonstrated that semen chilling induces changes in the sperm cell membrane and affects sperm motility (ConejoNava et al., 2003). Boar semen is particularly sensitive to chilling shock. That is why the storage temperature is important. Boar spermatozoa are more sensitive to the effect of low temperature than sperm cells of males of other animal species due to a different composition of sperm cell membrane phospholipids (De Leeuw et al., 1990). The data presented in this work show that the sperm motility of boars producing highly sensitive semen (group I) diminishes at a much higher rate during storage than the motility of spermatozoa of boars providing less sensitive semen. This argues for the usefulness of measuring sperm motility changes during semen storage as a criterion of insemination boar selection accuracy.

In the present study we also concluded that a low frequency of secondary morphological changes, not increasing during semen storage, can also be an index which can confirm the accuracy of the selection of males for insemination purposes. This seems obvious, since the boars were selected for the groups according to the criterion of secondary morphological change incidence in the spermatozoa. It should be noted, though, that semen storage did not cause an increase in the incidence of secondary morphological changes in the group of boars considered to provide sperm of low sensitivity. On the other hand, with continued storage there was a marked increase in the incidence of spermatozoa with secondary morphological changes in the group of boars regarded as producing more sensitive semen. Greater semen sensitivity is then manifested in an increasing incidence of secondary morphological changes in spermatozoa during semen storage. This is very important, since sperm structure and morphology affects fertilisation efficacy and embryo development (Didion et al., 2009).

In this study we pointed out the importance of the incidence of spermatozoa with undamaged cell membranes as an index which confirms the accuracy of insemination male selection. The semen of group I boars (higher semen sensitivity) was found to contain more spermatozoa with damaged cell membranes in comparison with group II boars. Cell membrane integrity is undoubtedly connected with sperm quality and fertilisation capacity. This is confirmed by observations made by Foster et al. (2011) and Love (2012). The importance of cell membrane integrity evaluation for assessments of sperm sensitivity lies in the 
fact that plasmolemma is vulnerable to external factors. The cell membrane of boar spermatozoa is particularly sensitive to temperature changes. A high polyunsaturated fatty acid content causes the cell membrane of boar spermatozoa to be more sensitive to damage than cell membranes of males of other species. Due to this, boar spermatozoa are highly sensitive to oxidative stress (Agarwal et al., 2008). The importance of cell membrane integrity for assessing sperm sensitivity stems from transformations of lipids which are an important component of the sperm cell membrane. When semen is chilled, the lipid fractions become separated. Sperm cell membrane permeability rises then. The structure of cell membrane lipids has a variable content of unsaturated fatty lipids and cholesterol. This variability is associated with the traits of individual boars (Waterhouse et al., 2006). That is why it can be a factor in differentiating the sensitivity of semen from particular sires. Owing to such differences, spermatozoa of some sires are more resistant to the effects of semen processing techniques, such as: dilution, mixing, preservation and chilling. It was probably for this reason that the less sensitive semen had a much lower frequency of spermatozoa with damaged cell membranes. The temperature of $12^{\circ} \mathrm{C}$ is considered to be the critical temperature level for boar semen (Althouse et al., 1998). Diluted semen is usually stored at $17^{\circ} \mathrm{C}$. This was also the temperature at which the semen analysed in this study was stored. The temperature of storage for boar semen should remain within the range of $15^{\circ} \mathrm{C}$ to $20^{\circ} \mathrm{C}$ (Shimatsu et al., 2002). Such temperature is regarded as optimal for storing boar semen. Sperm metabolism and the secretion of lactic acid and other metabolites, specific to semen storage, is limited then (Shimatsu et al., 2002). However, the data of this study show that semen storage is associated with significant changes in sperm motility and cell membrane integrity which are far more pronounced in highly sensitive semen.

No changes in the $\mathrm{pH}$ of the semen and in the sperm chromatin structure were observed in this study during semen storage. This suggests that these indices are little useful for the purpose of confirming the accuracy of selection of insemination boars. Sperm chromatin structure damage is more likely associated with the incidence of spermatozoa with primary changes (Enciso et al., 2011). Chromatin structure anomalies usually appear during spermiogenesis (Andrabi, 2007). It is then that changes in nuclear protein composition occur as a result of which histones are replaced with protamins. These changes are associated with a transformation of sperm chromatin structure and it is at this stage that anomalies appear. The data of this study show that chromatin structure changes in semen regarded as sensitive do not take place more often than in the semen of boars producing ejaculates of low sensitivity.
Opinions concerning chromatin structure and its association with sperm morphology and the capacity of spermatozoa to fertilise the egg cell are divided. Some studies suggest a relationship between sperm morphology and chromatin instability incidence (Kim et al., 2013) or incorrect chromatin structure (Fischer et al., 2003), while others do not confirm such interrelation (Khalili et al., 2006). Most tests evaluating sperm chromatin are based on the use of fluorescent dyes. Fluorescence dye staining does not enable a simultaneous evaluation of chromatin structure and sperm morphology. Therefore, it seems well-founded to use such sperm staining techniques to allow simultaneously determining the structure of sperm head chromatin and sperm morphology.

In conclusion, it should be stated that the semen of particular males differs in the level of its sensitivity to the influence of factors associated with the preparation of sperm for insemination and sperm storage before insemination interventions. Sperm sensitivity to external factors is an individual male trait and it is possible to select individual males that produce semen with low sensitivity to pre-insemination procedures. The incidence of secondary morphological changes in spermatozoa, defined at the beginning of insemination service of a given boar, is a simple and practically feasible index of boar semen sensitivity. The assessment of secondary morphological change incidence can be used as a tool in the selection of boars for their insemination service fitness. Factors that confirm the accuracy of insemination male selection can include a low rate of sperm motility decrease during the storage of diluted semen, low and contained incidence of secondary morphological changes in spermatozoa during semen storage (preferably up to 5\%) and a high frequency of spermatozoa with undamaged cell membranes. Measurements of $\mathrm{pH}$ changes occurring during semen storage and the assessment of the state of sperm chromatin structure are of little use for the confirmation of the accuracy of insemination boar selection.

\section{CONFLICT OF INTEREST}

We certify that there is no conflict of interest with any financial organization regarding the material discussed in the manuscript.

\section{ACKNOWLEDGMENTS}

This work has been prepared as a part of the project granted by Polish Government provided by the National Centre for Research and Development Nr N R12 0013 06/2009. 


\section{REFERENCES}

Agarwal, A., K. Makker, and R. Sharma. 2008. Clinical relevance of oxidative stress in male factor infertility: an update. Am. J. Reprod. Immunol. 59:2-11.

Althouse, G. C., M. E. Wilson, C. Kuster, and M. Parsley. 1998. Characterization of lower temperature storage limitations of fresh-extended porcine semen. Theriogenology 50:535-543.

Andrabi, S. M. 2007. Mammalian sperm chromatin structure and assessment of DNA fragmentation. J. Assist. Reprod. Genet. 24:561-569.

Blom, E. 1981. The morphological estimation of the spermatozoa defects of bull II. The proposal of new classification of spermatozoa defects. Med. Weter. 37:239-242.

Bochenek, M., Z. Smorąg, and Z. Pilch. 2001. Sperm chromatin structure assay of bulls qualified for artificial insemination. Theriogenology 56:557-567.

Briz, M. D., S. Bonet, B. Pinart, and R. Camps. 1996. Sperm malformations throughout the boar epididymal duct. Anim. Reprod. Sci. 43:221-239.

Broekhuijse, M. L., H. Feitsma, and B. M. Gadella. 2012. Artificial insemination in pigs: predicting male fertility. Vet. Q. 32:151-157.

Conejo-Nava, J., R. Fierro, C. G. Gutierrez, and M. Betancourt. 2003. Membrane status and in vitro capacitation of porcine sperm preserved in long-term extender at 16 degrees C. Arch. Androl. 49:287-295.

De Ambrogi, M., J. Ballester, F. Saravia, I. Caballero, A. Johannisson, M. Wallgren, M. Andersson, and H. RodriguezMartinez. 2006. Effect of storage in short and long-term commercial semen extenders on the motility, plasma membrane and chromatin integrity of boar spermatozoa. Int. J. Androl. 29:543-552.

De Leeuw, F. E., B. Colenbrander, and A. J. Verkleij. 1990. The role membrane damage plays in cold shock and freezing injury. Reprod. Domest. Anim. 1:95-104.

Didion, B. A., K. M. Kasperson, R. L. Wixon, and D. P. Evenson. 2009. Boar fertility and sperm chromatin structure status: A retrospective report. J. Androl. 30:655-660.

Enciso, M., H. Cisale, S. D. Johnston, J. Sarasa, J. L. Fernández, and J. Gosálvez. 2011. Major morphological sperm abnormalities in the bull are related to sperm DNA damage. Theriogenology 76:23-32.

Fischer, M. A., J. Willis, and A. Zini. 2003. Human sperm DNA integrity: correlation with sperm cytoplasmic droplets. Urology 61:207-211.

Foster, M. L., C. C. Love, D. D. Varner, S. P. Brinsko, K. Hinrichs, S. Teague, K. Lacaze, and T. L. Blanchard. 2011. Comparison of methods for assessing integrity of equine sperm membranes. Theriogenology 76:334-341.

Kawęcka, M., A. Pietruszka, E. Jacyno, R. Czarnecki, and M. Kamyczek. 2008. Quality of semen of young boars of the breeds Pietrain and Duroc and their reciprocal crosses. Arch. Tierz. 51:42-54.
Khalili, M. A., F. Aghaie-Maybodi, M. Anvari, and A. R. Talebi. 2006. Sperm nuclear DNA in ejaculates of fertile and infertile men: correlation with semen parameters. Urol. J. 3:154-159.

Kim, H. S., M. J. Kang, S. A. Kim, S. K. Oh, H. Kim, S. Y. Ku, S. H. Kim, S. Y. Moon, and Y. M. Choi. 2013. The utility of sperm DNA damage assay using toluidine blue and aniline blue staining in routine semen analysis. Clin. Exp. Reprod. Med. 40:23-28.

Knox, R. V. and B. M. Yantis. 2014. The effect of numbers of frozen-thawed boar sperm and addition of prostaglandin F2 $\alpha$ at insemination on fertility in pigs. Anim. Reprod. Sci. 151:194200.

Kondracki, S., M. Iwanina, A. Wysokińska, and M. Huszno. 2012. Comparative analysis of Duroc and Pietrain boar sperm morphology. Acta Vet. Brno 81:195-199.

Kwon, W. S., M. S. Rahman, J. S. Lee, J. Kim, S. J. Yoon, Y. J. Park, Y. A. You, S. Hwang, and M. G. Pang. 2014. A comprehensive proteomic approach to identifying capacitation related proteins in boar spermatozoa. BMC Genomics 15:897.

López Rodríguez, A., T. Rijsselaere, P. Vyt, A. Van Soom, and D. Maes. 2012. Effect of dilution temperature on boar semen quality. Reprod. Domest. Anim. 47:e63-e66.

Love, C. C. 2012. Measurement of concentration and viability in stallion sperm. J. Equine Vet. Sci. 32:464-466.

Manee-In, S., S. Parmornsupornvichit, S. Kraiprayoon, T. Tharasanit, P. Chanapiwat, and K. Kaeoket. 2014. L-carnitine supplemented extender improves cryopreserved-thawed cat epididymal sperm motility. Asian-Australas. J. Anim. Sci. 27:791-796.

Martín-Hidalgo, D., F. J. Barón, A. Robina, M. J. Brygado, A. H. Llera, L. J. García-Marín, and M. C. Gil. 2013. Inter- and intra-breed comparative study of sperm motility and viability in Iberian and Duroc boar semen during long-term storage in MR-A and XCell extenders. Anim. Reprod. Sci. 139:109-114.

Oberlender, G., L. D. Murgas, M. G. Zangeronimo, A. C. Silva, L. J. Pereira, and R. A. Muzzi. 2012. Comparison of two different methods for evaluating boar semen morphology. Arch. Med. Vet. 44:201-205.

Park, Y. J., W. S. Kwon, S. A. Oh, and M. G. Pang. 2012. Fertilityrelated proteomic profiling bull spermatozoa separated by percoll. J. Proteome Res. 11:4162-4168.

Shimatsu, Y., M. Uchida, R. Niki, and H. Imai. 2002. Liquid storage of miniature boar semen. Exp. Anim. 51:143-147.

Waberski, D., A. M. Petrunkina, and E. Töpfer-Petersen. 2008. Can external quality control improve pig AI efficiency? Theriogenology 70:1346-1351.

Waterhouse, K. E., P. O. Hofmo, A. Tverdal, and J. R. Miller. 2006. Within and between breed differences in freezing tolerance and plasma membrane fatty acid composition of boar sperm. Reproduction 131:887-894.

Wysokińska, A. and S. Kondracki. 2014. Assessment of changes in sperm cell membrane integrity occurring during the storage of semen from genetically different males using two diagnostic methods. Can. J. Anim. Sci. 94:601-606. 